\title{
Variable anatomy of the right coronary artery supply to the left ventricle
}

\author{
JILL ADAMS, TOM TREASURE
}

From the Department of Anatomy, Middlesex Hospital Medical School, and the Cardiothoracic Surgical Unit, Middlesex Hospital, London

ABSTRACT The right coronary artery is "dominant" (contributing to the supply of the left ventricular muscle) in $90 \%$ of people. Although the most common pattern is for the right coronary artery to bifurcate at the crux giving the posterior descending (posterior interventricular) artery, a branch may arise before the crux, either as an aberrant acute marginal artery or as an early posterior descending artery, crossing the diaphragmatic surface of the right ventricle. Recognition of this possibility may be important if surgical revascularisation is to be complete. To establish the frequency of these different patterns of blood supply, 22 hearts were studied in the dissecting room, and 100 consecutive coronary angiograms and 100 consecutive operative drawings were reviewed. A vessel arising before the crux contributed to the supply of the left ventricle in one third of cases.

Standard anatomical texts state that the posterior descending (posterior interventricular) coronary artery, one of the terminal branches of the right coronary artery, is given off at the crux and then runs in the inferior interventricular groove, supplying the posterior part of the septum and the adjacent left ventricle (fig 1a). In his classic study' James noted that the posterior descending artery sometimes leaves the right coronary artery before the crux and courses across the inferior surface of the right ventricle before reaching the interventricular groove (fig $1 b)$. With increasing familiarity with the detailed anatomy gained in the course of investigation and surgery for coronary artery disease, it has been noticed that this is not a rare occurrence and in extreme examples the virtually constant right coronary artery branch arising at the acute margin of the heart (the acute marginal artery) departs from its usual course to reach the distal part of the interventricular groove (fig 1c). We are not, however, aware of any account of the frequency of these variations.

The correlation of angiographic appearance and three dimensional anatomy, with the ability to predict accurately the position of diseased vessels, is important in coronary artery surgery, especially now that the trend is towards grafting more coronary

Address for reprint requests: Mr $\mathrm{T}$ Treasure, Cardiothoracic Surgical Unit, Middlesex Hospital, London W1N 8AA.

Accepted 11 March 1985 branches. The aim of this study was to quantify the occurrence of this variability in the right coronary artery supply to the left ventricular muscle.

\section{Methods}

A consecutive series of 100 coronary angiograms was reviewed and a decision was made whether the right coronary artery was "dominant" (that is, contributed a coronary artery supply to the left ventricle) and, if so, whether the main vessel that reached the interventricular groove arose at the crux, at the acute margin, or in an intermediate position. An intermediate position was defined as at least one third of the way from the crux to the acute margin (fig 2).

In all coronary artery operations performed the notes included a diagram of the vessels, derived from the coronary angiogram and modified in the light of operative findings. The findings in a consecutive series of 100 operations were analysed and the posterior descending artery was classified according to whether it arose at the crux or earlier. Origin at the acute margin or from an intermediate position had not always been clearly identified by the surgeon, so this distinction was not made in this group; but an "early" branch was at least one third of the way from the crux as before.

In the anatomy department 22 unselected hearts were dissected to expose all coronary artery 618 

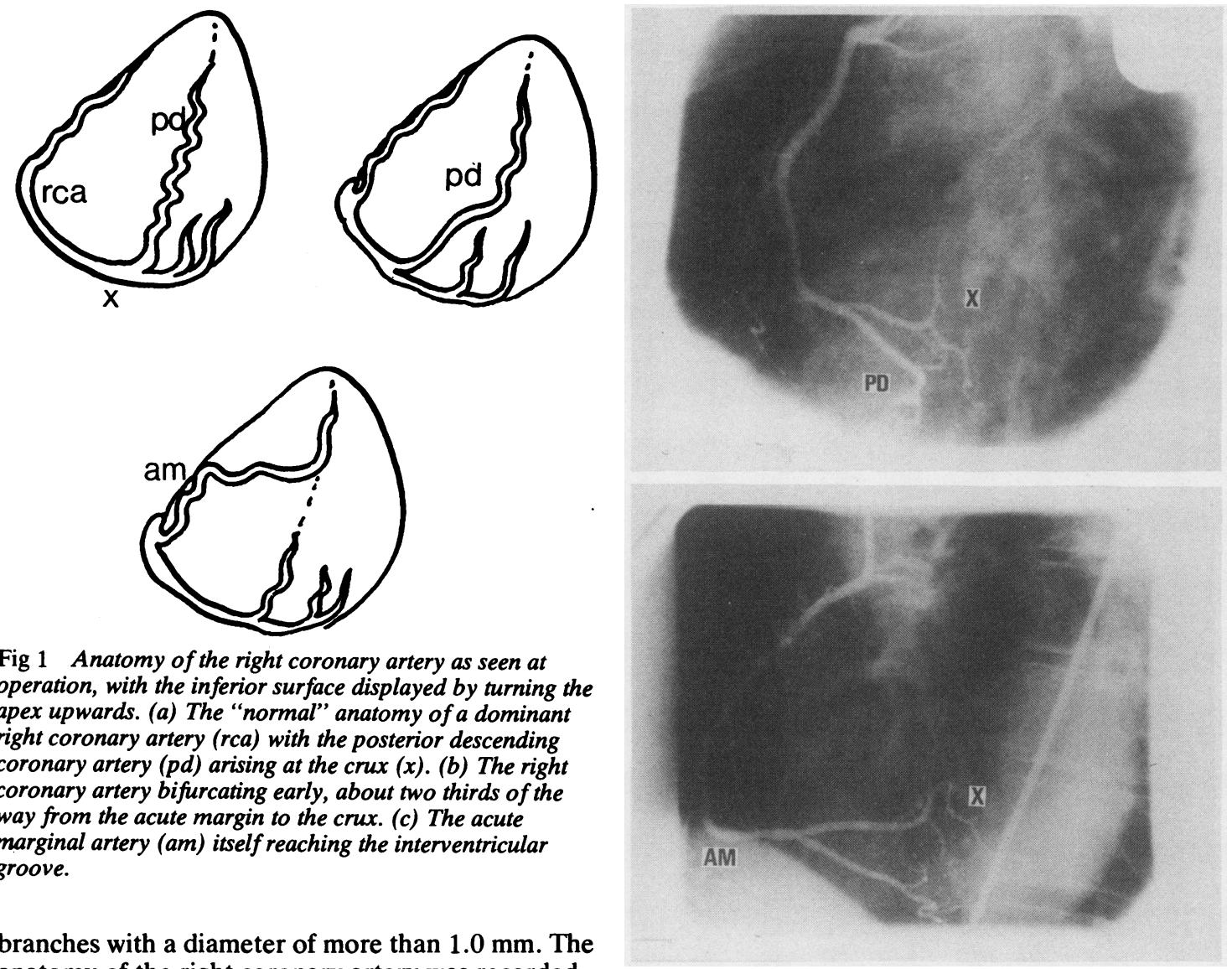

branches with a diameter of more than $1.0 \mathrm{~mm}$. The anatomy of the right coronary artery was recorded.

\section{Results}

The results are shown in the table. While a dominant left coronary artery, as expected, was uncommonthe proportion being not significantly different $(\mathrm{p}>$ 0.05 ) from the usually quoted $10 \%$-an "early" posterior descending artery, arising before the crux, was found in $32 \%$ of angiograms and operative drawings and $36 \%$ of dissections.

Fig 2 Angiogram of a right coronary artery in (a) the left anterior oblique and $(b)$ the anteroposterior projection. The artery to the atrioventricular node running vertically in the septum identifies the crux $(X)$. The posterior descending artery $(P D)$ arises early and leaves the atrioventricular groove about one third of the way between the acute margin $(A M)$ and the crux $(X)$.

\section{Discussion}

The overall proportion of non-dominant right coro-

Coronary artery patterns seen in angiograms, at operation, and in dissections

\begin{tabular}{llcc}
\hline & \multicolumn{2}{c}{$\%$ of cases $(70 \%$ confidence limits) } & Dissections $(n=22)$ \\
\cline { 2 - 4 } & \multicolumn{1}{c}{ Angiograms $(n=100)$} & Operations $(n=100)$ & $14(7-26)$ \\
\hline Dominant left coronary artery & $11(8-15)$ & $5(3-9)$ & $50(37-63)$ \\
PDA at crux & $57(51-62)$ & $63(57-68)$ & $36(25-50)$ \\
Early PDA & $32(27-38)$ & $32(27-38)$ & 27 \\
$\begin{array}{l}\text { At acute margin } \\
\text { Intermediate }\end{array}$ & 13 & & 9 \\
Total & 19 & 100 & 100 \\
\hline
\end{tabular}

PDA-posterior descending artery. 
nary artery systems was much as expected. A posterior descending artery arising before the crux was found in about one third of cases in all the groups. About half of these arose in an intermediate position, a relatively minor anatomical variation that is well recognised by surgeons performing coronary artery operations. Those that originated at the acute margin (fig $1 c$ ) represent a greater deviation from normal; six of the eight "early" posterior descending arteries in the dissected hearts were of this type. The vessel may be large and supply a substantial area of left ventricular muscle or communicate with other systems and thus merit grafting. Awareness of its presence and anatomy may be important in some instances to achieve complete revascularisation.

We are greatful to Professor Bernard Wood for allowing us to study hearts in the anatomy department and for his help and advice.

\section{Reference}

1 James TN. Anatomy of the coronary arteries. New York: Heber, 1961. 